\title{
Indirect probe of electroweakly interacting particles at the high-luminosity Large Hadron Collider
}

\author{
Shigeki Matsumoto, Satoshi Shirai and Michihisa Takeuchi \\ Kavli Institute for the Physics and Mathematics of the Universe (WPI), \\ The University of Tokyo Institutes for Advanced Study, The University of Tokyo, \\ Kashiwa 277-8583, Japan \\ E-mail: shigeki.matsumoto@ipmu.jp, satoshi.shirai@ipmu.jp, \\ michihisa.takeuchi@ipmu.jp
}

ABSTRACT: Many extensions of the standard model (SM) involve new massive particles charged under the electroweak gauge symmetry. The electroweakly interacting new particles affect various SM processes through radiative corrections. We discuss the possibility of detecting such new particles based on the precise measurement of the SM processes at high energy hadron colliders. It then turns out that Drell-Yan processes receive radiative corrections from the electroweakly interacting particles at the level of $\mathcal{O}(0.1-10) \%$. It is hence possible to indirectly search for the Higgsino up to the mass of $400 \mathrm{GeV}$ and the quintet (5-plet) Majorana fermion up to the mass of $1200 \mathrm{GeV}$ at the high-luminosity running of the Large Hadron Collider, if the systematic uncertainty associated with the estimation of the SM background becomes lower than the statistical one.

KeYwords: Beyond Standard Model, Supersymmetric Standard Model

ARXIV EPRINT: 1711.05449 


\section{Contents}

1 Introduction 1

2 Radiative correction from EWIMP 3

3 Analysis of collider signal $\quad 6$

3.1 Fitting based search 6

$\begin{array}{lll}3.2 & \text { MC based search } & 7\end{array}$

3.3 Capability of EWIMP detection at LHC 8

4 Conclusion and discussion $\quad 9$

\section{Introduction}

Various types of electroweakly interacting massive particles (EWIMPs) are introduced in new physics models beyond the standard model (SM). For instance, in supersymmetric (SUSY) extensions of the SM, almost all supersymmetric partners of SM particles are charged under the electroweak symmetry, namely $\mathrm{SU}(2)_{\mathrm{L}} \times \mathrm{U}(1)_{\mathrm{Y}}$ gauge symmetry. This is also the case for other new physics models such as extra dimension models, extended Higgs models and so on. Such EWIMPs often play significant roles for the origin of the electroweak symmetry breaking and/or can be excellent candidates for dark matter in our universe.

The latter role is particularly interesting, as the electroweak interaction makes the dark matter satisfying the weakly interacting massive particle (WIMP) hypothesis and visible at direct and indirect dark matter detection experiments. One of such candidates is the wino dark matter in SUSY having the electroweak quantum number of $\mathbf{3}_{0}$, and it has actually rich dark matter signatures thanks to the interaction [1-7]. It is also worth pointing out that the wino dark matter is motivated very well from the viewpoint of particle physics theory; it is a generic prediction from SUSY SMs with the anomaly mediation $[8,9]$. This framework is known to be compatible with the so-called "mini-split SUSY" scenario [10-15], and the discovery of the $125 \mathrm{GeV}$ Higgs boson $[16,17]$ triggers the framework to attract more and more attention [18-24]. In fact, detailed phenomenological studies on the wino dark matter are stimulated in many studies [25-28]. Another interesting candidate is the dark matter having a large electroweak quantum number, because such a quantum number makes the dark matter particle stable without imposing any ad hoc dark matter parity. The quintet fermion whose quantum number is $\mathbf{5}_{0}$ and the septet scalar having the quantum number of $\mathbf{7}_{0}$ are such examples. Those are now referred as the "minimal dark matter" [29-31]. There also be an interesting candidate motivated from the electroweak symmetry breaking; the Higgsino having the electroweak quantum 
number of $\mathbf{2}_{ \pm 1 / 2}$, which is often predicted to be the lightest SUSY particle in the so-called natural SUSY scenario [32]. Interestingly, its mass is required to be smaller than $350 \mathrm{GeV}$ to obtain the electroweak scale naturally $[33,34]$, which is kinematically accessible at the Large Hadron Collider (LHC). Discovery and measurement of EWIMP at colliders are thus a crucial test for physics beyond the SM.

Collider signals of EWIMP is strongly model-dependent. For instance, it is crucial whether or not the EWIMP is also charged under the $\mathrm{SU}(3)_{\mathrm{C}}$ symmetry for its production at hadron colliders. Moreover, collider signatures are strongly affected by whether and how the EWIMP decays. The search for the EWIMP at hadron colliders becomes difficult in general if its decay products are very soft [35-43]. A prominent example is the direct production of the electroweakly interacting dark matter. Though the search for the large missing energy accompanied with high $P_{\mathrm{T}}$ jets or photons is conventionally used to search for the EWIMP, it does not work efficiently due to huge SM backgrounds as well as the small production cross section of the EWIMP. In some cases, the mass difference among the $\mathrm{SU}(2)_{\mathrm{L}}$ multiplet becomes so small that its charged component is long-lived. When the charged track caused by the long-lived particle is detectable, it may overcome the huge SM backgrounds. ${ }^{1}$ This is, however, not a generic feature of EWIMP. Even if we consider EWIMP dark matter, its coupling to the Higgs field may enhance the mass difference, making the decay length of the charged component too short [58-60]. It is therefore important to develop an independent method for the EWIMP search which does not rely on the charged track search.

We consider an indirect probe of EWIMP through its radiative corrections to SM processes. We particularly focus on the dilepton production by the Drell-Yan process at the LHC; the EWIMP is expected to modify the lepton invariant mass distribution $\left(m_{\ell \ell}\right)$. It is known that, when $m_{\ell \ell}$ is much smaller than twice the EWIMP mass $(m)$, the correction is effectively described by dimension-six operators and is proportional to $m_{\ell \ell}^{2} / m^{2}$. On the other hand, the EWIMP affects the running of the electroweak gauge couplings when $m_{\ell \ell} \gg 2 m$, leading to the correction proportional to $\ln \left(m_{\ell \ell}^{2} / m^{2}\right)$ [61-63]. In this paper, we show that the correction becomes an extremum of $\mathcal{O}(0.1-10) \%$ when $m_{\ell \ell} \simeq 2 m$, making the EWIMP detectable at the LHC utilizing this feature. We find that the present observation of the dilepton mass distribution already excludes the EWIMP having a large electroweak quantum number. It will be possible to search for the EWIMP having a smaller quantum number at the high-luminosity LHC (HL-LHC), so that the indirect EWIMP search becomes as important as the direct production search with mono$X$ and missing energy in the future.

\footnotetext{
${ }^{1}$ In fact, the most sensitive search for the pure wino dark matter is based on this strategy [44-49], as the charged wino has a decay length of about $6 \mathrm{~cm}[50,51]$. Furthermore, it has been shown recently that a much shorter decay length becomes detectable by improving the track reconstruction and/or modifying the detector $[52,53]$. It is then even possible to detect the almost pure Higgsino whose charged component has a decay length shorter than $1 \mathrm{~cm}$. The strategy based on the charged track is also applicable to the case that EWIMP is not dark matter but a coannihilating partner like the gaugino coannihilation [54-56]; the search for the EWIMP becomes powerful despite its soft decay products when it is long-lived enough [57]
} 


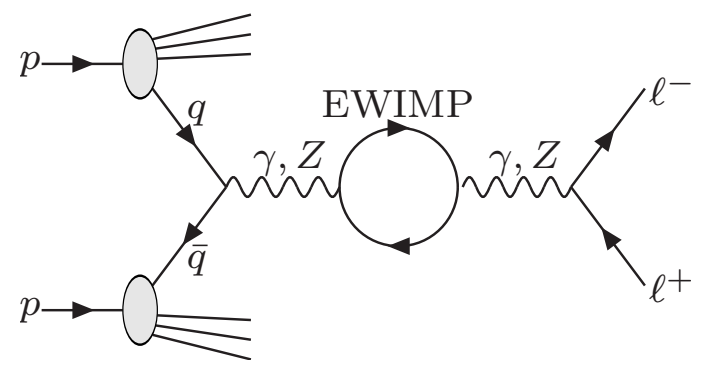

Figure 1. Radiative correction from the electroweakly interacting massive particle (EWIMP).

\section{Radiative correction from EWIMP}

We focus on the Drell-Yan process $p p \rightarrow \ell^{+} \ell^{-}+X$ in this paper with $p$ and $\ell^{ \pm}$being a proton and a lepton, and discuss how EWIMP modifies the lepton invariant mass distribution $m_{\ell \ell}$ at high energy hadron colliders. The EWIMP affects the differential cross section of the process through the loop correction shown in figure 1. After integrating the EWIMP field out from the original Lagrangian at one-loop level, we obtain the following effective Lagrangian: ${ }^{2}$

$$
\mathcal{L}_{\mathrm{eff}}=\mathcal{L}_{\mathrm{SM}}+\kappa \frac{g^{2} C_{W W}}{8} W_{\mu \nu}^{a} \Pi\left(-D^{2} / m^{2}\right) W^{a \mu \nu}+\kappa \frac{g^{\prime 2} C_{B B}}{8} B_{\mu \nu} \Pi\left(-\partial^{2} / m^{2}\right) B^{\mu \nu}+\cdots,
$$

where $\mathcal{L}_{\mathrm{SM}}$ is the SM Lagrangian, $m$ is the EWIMP mass, $g\left(g^{\prime}\right)$ is the gauge coupling of $\mathrm{SU}(2)_{\mathrm{L}}\left(\mathrm{U}(1)_{\mathrm{Y}}\right)$ and $W_{\mu \nu}^{a}\left(B_{\mu \nu}\right)$ is the corresponding field strength tensor, respectively, with $D$ being the covariant derivative acting on $W^{a \mu \nu}$. Parameters $C_{W W}$ and $C_{B B}$ are defined as $\boldsymbol{C}_{\boldsymbol{W} \boldsymbol{W}} \equiv \boldsymbol{\xi}\left(\boldsymbol{n}^{\mathbf{3}}-\boldsymbol{n}\right) / \mathbf{6}$ and $\boldsymbol{C}_{\boldsymbol{B} \boldsymbol{B}} \equiv \mathbf{2} \boldsymbol{\xi} \boldsymbol{n} \boldsymbol{Y}^{\mathbf{2}}$ for the SU(2) $)_{\mathrm{L}}$ n-tuplet EWIMP having the hypercharge $Y$ and the color degree of freedom $\xi$, while $\kappa$ takes a value of $1 / 2,1,4$ and 8 when the EWIMP is a real scalar, complex scalar, Majorana and Dirac fermions, respectively. The ellipsis in the Lagrangian includes operators composed of the strength tensors more than two, but those are not relevant to the following discussion. The function $\Pi(x)$ is the renormalized self-energy of the electroweak gauge bosons from the EWIMP loop:

$$
\Pi(x)= \begin{cases}\frac{1}{16 \pi^{2}} \int_{0}^{1} d y y(1-y) \ln \left[1-y(1-y) x-i 0^{+}\right] & \text {(Fermion), } \\ \frac{1}{16 \pi^{2}} \int_{0}^{1} d y(1-2 y)^{2} \ln \left[1-y(1-y) x-i 0^{+}\right] & \text {(Scalar). }\end{cases}
$$

We have used the $\overline{\mathrm{MS}}$ regularization scheme with the renormalization scale of $\mu=m$.

All the effect of the EWIMP on the process is encoded in the operators involving two field strength tensors. When the EWIMP mass is much larger than the partonic collision energy $\hat{s}^{1 / 2}$, namely $m^{2} \gg-\partial^{2}=\hat{s}$, the operators give dimension-six ones, $\left(D_{\mu} W^{a \mu \nu}\right)\left(D^{\rho} W_{\rho \nu}^{a}\right)$ and $\left(\partial_{\mu} B^{\mu \nu}\right)\left(\partial^{\rho} B_{\rho \nu}\right)$. In contrast, the function $\Pi$ behaves as

\footnotetext{
${ }^{2}$ We only take the electroweak gauge interactions of the EWIMP into account for simplicity and neglect other renormalizable interactions (such as the Yukawa interaction, etc.) to derive the effective Lagrangian.
} 
$\Re\left[\Pi\left(-\partial^{2} / m^{2}\right)\right] \sim \log \left(\hat{s} / m^{2}\right)$ when $m^{2} \ll-\partial^{2}=\hat{s}$, which is eventually translated into the running effect of the electroweak gauge couplings. The effect of the EWIMP in these two extreme regions has already been studied in several papers [61-63]. On the other hand, we use the effective Lagrangian in eq. (2.1) directly, as we are interested in the effect at the region around $s \sim 4 m^{2}$.

The matrix element of the Drell-Yan process is obtained from the effective Lagrangian. Leading order (LO) contribution is from SM interactions and its explicit form is

$$
\mathcal{M}_{\mathrm{LO}}\left[q(p) \bar{q}\left(p^{\prime}\right) \rightarrow \ell^{-}(k) \ell^{+}\left(k^{\prime}\right)\right]=\sum_{V=\gamma, Z} \frac{\left[\bar{v}\left(p^{\prime}\right) \gamma^{\mu} \Gamma_{q}^{V} u(p)\right]\left[\bar{u}(k) \gamma_{\mu} \Gamma_{l}^{V} v\left(k^{\prime}\right)\right]}{\hat{s}-m_{V}^{2}},
$$

where $\Gamma_{f}^{Z}=g_{Z}\left(v_{f}-a_{f} \gamma_{5}\right)$ and $\Gamma_{f}^{\gamma}=e Q_{f}$, while $g_{Z}=g / c_{W}$ and $e=g s_{W}$, where $s_{W}=$ $\sin \theta_{W}$ and $c_{W}=\cos \theta_{W}$ with $\theta_{W}$ being the weak mixing angle. Coefficients $\left(v_{f}, a_{f}, Q_{f}\right)$ are $\left(1 / 4-2 s_{W}^{2} / 3,1 / 4,2 / 3\right),\left(-1 / 4+s_{W}^{2} / 3,-1 / 4,-1 / 3\right)$ and $\left(-1 / 4+s_{W}^{2},-1 / 4,-1\right)$ for up-type quarks, down-type quarks and charged leptons, respectively. The mass of the electroweak gauge boson is given by $m_{V}$, while the center-of-mass energy at this partonlevel process is denoted by $\hat{s}^{1 / 2}$. Next leading order contribution (NLO) to the matrix element from the EWIMP loop diagram shown in figure 1 is given by the following formula:

$\mathcal{M}_{\mathrm{BSM}}\left[q(p) \bar{q}\left(p^{\prime}\right) \rightarrow \ell^{-}(k) \ell^{+}\left(k^{\prime}\right)\right]=\sum_{V, V^{\prime}} \frac{d_{V V^{\prime}}\left[\bar{v}\left(p^{\prime}\right) \gamma^{\mu} \Gamma_{q}^{V} u(p)\right] \hat{s} \Pi\left(\hat{s} / m^{2}\right)\left[\bar{u}(k) \gamma_{\mu} \Gamma_{l}^{V^{\prime}} v\left(k^{\prime}\right)\right]}{\left(\hat{s}-m_{V}^{2}\right)\left(\hat{s}-m_{V^{\prime}}^{2}\right)}$,

where each coefficient $d_{V V^{\prime}}$ in the numerator is defined as $d_{Z Z}=\kappa\left(g_{Z}^{2} / 2\right)\left(c_{W}^{4} C_{W W}+\right.$ $\left.s_{W}^{4} C_{B B}\right), d_{\gamma \gamma}=\kappa\left(e^{2} / 2\right)\left(C_{W W}+C_{B B}\right)$ and $d_{Z \gamma}=d_{\gamma Z}=\kappa\left(e g_{Z} / 2\right)\left(c_{W}^{2} C_{W W}-s_{W}^{2} C_{B B}\right)$, respectively.

We show in figure 2 that how the EWIMP contribution modifies the lepton invariant mass distribution at the $13 \mathrm{TeV}$ LHC, where the difference between the differential cross sections of the Drell-Yan process with and without the EWIMP contribution (normalized by the SM prediction at LO) is shown as a function of the lepton invariant mass $m_{\ell \ell}=\hat{s}^{1 / 2}$ for three EWIMP cases; Wino (Majorana fermion with the quantum number of $3_{0}$ ), Higgsino (Dirac fermion with the quantum number of $2_{ \pm 1 / 2}$ ) and bosonic minimal dark matter (real scalar with the quantum number of $7_{0}$ ) with their masses fixed to be $300 \mathrm{GeV}$. The SM cross section $\hat{\sigma}_{\mathrm{SM}}$ is calculated at leading order. The cross section of the fermionic minimal dark matter (Majorana fermion with the quantum number of $5_{0}$ ) [29-31] is about five times larger than that of the wino. It can be seen that the modification becomes small when $m_{\ell \ell} \ll m$, while receives a logarithmic correction when $m_{\ell \ell} \gg m$, as expected from the discussion above. On the other hand, when $m_{\ell \ell} \sim 2 m$, the correction shows a characteristic feature due to the interference between LO and NLO contributions. The deviation becomes an (almost) extremum at $\hat{s}^{1 / 2}=2 m$ for a fermionic (scalar) EWIMP, which is analytically given by

$$
\frac{\hat{\sigma}_{\mathrm{BSM}}-\hat{\sigma}_{\mathrm{SM}}}{\hat{\sigma}_{\mathrm{SM}}} \simeq \Re\left[\frac{2 \mathcal{M}_{\mathrm{NLO}}}{\mathcal{M}_{\mathrm{LO}}}\right] \simeq \frac{\kappa g^{2}\left(n^{3}-n\right)}{6} \Pi(4)
$$




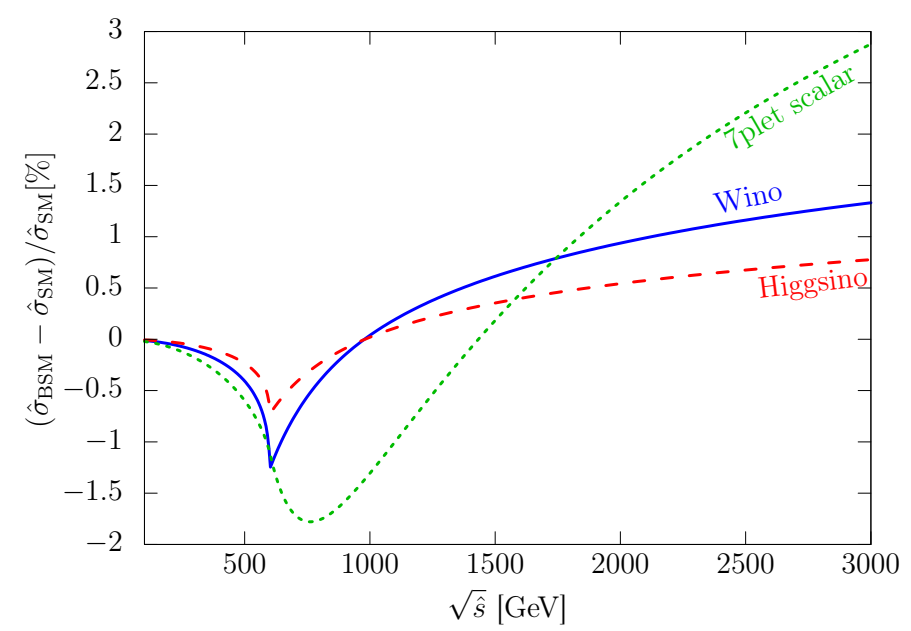

Figure 2. The difference between the differential cross sections of the Drell-Yan process with and without the EWIMP contribution (normalized by the SM prediction) at the $13 \mathrm{TeV}$ LHC as a function of $m_{\ell \ell}=\hat{s}^{1 / 2}$. Three EWIMP cases are depicted: Wino (Majorana fermion with the quantum number of $3_{0}$ ), Higgsino (Dirac fermion with the quantum number of $2_{ \pm 1 / 2}$ ) and bosonic minimal dark matter (real scalar with the quantum number of $7_{0}$ ) with their masses fixed to be $300 \mathrm{GeV}$.

where the effect of gauge boson mass and $\mathrm{U}(1)_{\mathrm{Y}}$ interaction is neglected. The loop function $\Pi(4)$ takes a value of $-1 /\left(36 \pi^{2}\right)$ for a fermionic EWIMP and $-1 /\left(72 \pi^{2}\right)$ for a scalar EWIMP. This characteristic feature is expected to be utilized to detect the EWIMP efficiently.

Note that the EWIMP affects the self-energy of the $Z$ boson at $p^{2}=m_{Z}^{2}$. To compensate this effect, we should shift the SM parameters properly to maintain the correct definitions of low-energy observables such as $G_{F}, \alpha$ and $m_{Z}$. The effect is, however, small for a heavy EWIMP with moderate gauge quantum numbers, for the EWIMP contribution to the self-energy is suppressed both by its mass and numerical factors: $16 \pi^{2} \Pi(x \rightarrow 0) \rightarrow-x / 30$. For instance, the wino with a mass of $100 \mathrm{GeV}$ affects the SM parameters only at the level of $\mathcal{O}(0.01) \%$. On the other hand, the wino affects the Drell-Yan process for $m_{\ell \ell} \gtrsim 2 m$ at $\mathcal{O}(1) \%$. We hence neglect the EWIMP effect on the modification of the SM parameters in following discussions.

Here, it is important to discuss the constraints from the the electroweak precision observables (EWPO) at the LEP, Tevatron and LHC experiments. It then turns out that the constraints on the EWIMP is not severe at present. For instance, a constraint is put by the measurement of the $W$ boson mass, which is obtained from the EWIMP oblique correction to various SM processes [64-67]. This correction is approximately given as follows:

$$
\delta m_{W} \simeq\left(\frac{m}{100 \mathrm{GeV}}\right)^{-2}\left(0.08 n\left(n^{2}-1\right)+0.3 n Y^{2}\right) \kappa \xi[\mathrm{MeV}]
$$

Since the uncertainty of the $W$ boson measurement is about $12 \mathrm{MeV}$ at present [68-70], the mass shift by the EWIMP contribution is not significant. Various EWPO constraints 


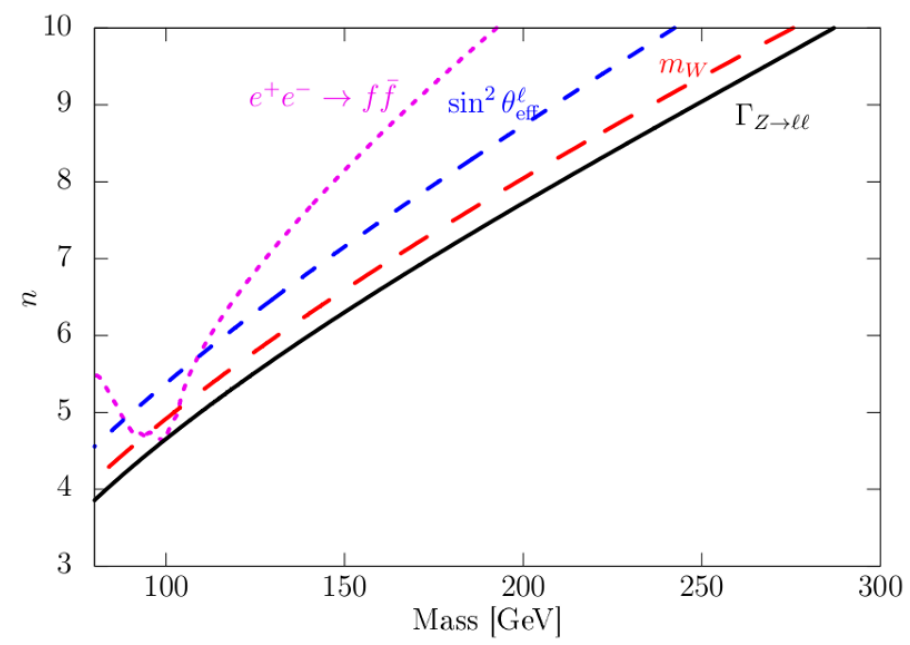

Figure 3. The current constraints on Majorana fermion with hypercharge zero from the electroweak precision measurements. The region above each line is excluded at $95 \%$ confidence level.

on a Majorana fermion with hypercharge zero are shown in figure 3 . The $\mathrm{SU}(2)_{\mathrm{L}}$ quantum number ' $n$ ' is formally treated as a real number to depict the figure. All the constraints are given at $95 \%$ confidence level, which are obtained from the measurement of the $W$ boson mass $m_{W}$ (observed value: $80.379 \pm 0.012 \mathrm{GeV} \& \mathrm{SM}$ prediction: $80.361 \pm 0.006 \mathrm{GeV}$ ), the partial decay width of the $Z$ boson into leptons $\Gamma_{Z \rightarrow \ell \ell}$ (observed value: $83.984 \pm$ $0.086 \mathrm{MeV} \& \mathrm{SM}$ prediction: $83.995 \pm 0.010 \mathrm{MeV})$, and the effective weak mixing angle $\sin ^{2} \theta_{\text {eff }}$ (observed value: $0.23153 \pm 0.00016 \&$ SM prediction: $0.23152 \pm 0.00005$ ) [70, 71] The constraint from the cross sections and the forward-backward asymmetries of various fermion pair production processes $e^{+} e^{-} \rightarrow f \bar{f}$ at the LEP II experiment [72] is also shown in the figure.

\section{Analysis of collider signal}

We discuss here the detection capability of EWIMP by measuring the dilepton distribution at the LHC, where $36 \mathrm{fb}^{-1}$ data at the $13 \mathrm{TeV}$ running is mainly used in our analysis [73]. We consider two different methods to deal with the $\mathcal{O}(0.1-1) \%$ deviation from the SM background. One is the "fitting based search", for the EWIMP contributes to the distribution destructively, as we have seen in the previous section. The other one is based on the background estimation through the Monte-Carlo simulation. The simulation now reproduces the observed data very well, so that the EWIMP contribution will be efficiently searched for through the likelihood test of the "EWIMP signal + SM background" hypothesis.

\subsection{Fitting based search}

The analysis is essentially the same as the conventional bump search at dilepton channels. Since the SM background is expected to give a smooth distribution on the channels and the observed data shows such a smooth distribution too, we can estimate the SM background 


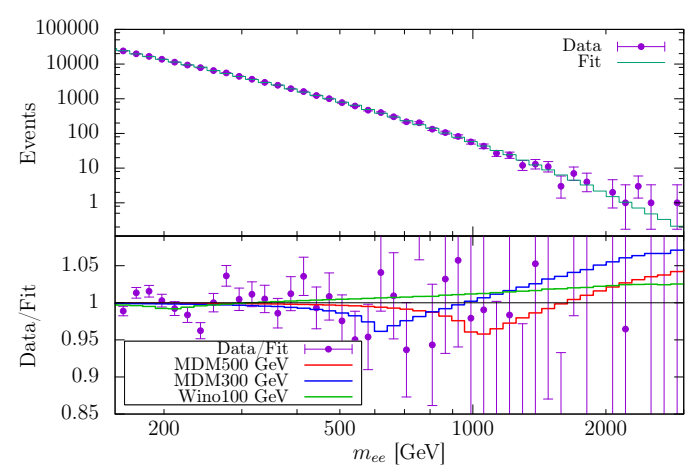

(a) Dielectron channel

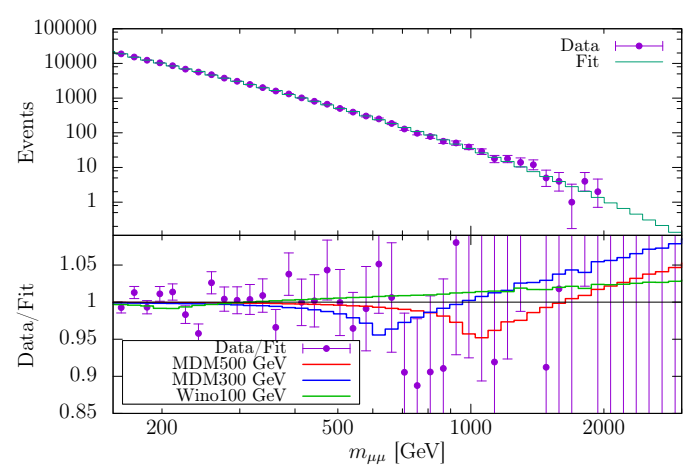

(b) Dimuon channel

Figure 4. Fitting the observational data by the SM background based on the function in eq. (3.1) for (a) dielectron and (b) dimuon channels with $m_{\ell \ell}$ being the lepton invariant mass. The data of $36 \mathrm{fb}^{-1}$ at the $13 \mathrm{TeV}$ LHC is used. Bottom panel in each figure shows the ratio between the data and the background as well as expected signals of the wino with the mass of $100 \mathrm{GeV}$ and the fermionic (5-tuplet) minimal dark matter (MDM) with its mass fixed to be $300 \mathrm{GeV}$ and $500 \mathrm{GeV}$.

in a data-driven way, namely by fitting the data using the following function [74]:

$$
\frac{d N_{\mathrm{BG}}}{d m_{\ell \ell}}=p_{1}(1-x)^{p_{2}} x^{p_{3}+p_{4} \log (x)+p_{5} \log ^{2}(x)},
$$

where $N_{\mathrm{BG}}$ is the leptonic invariant mass $\left(m_{\ell \ell}\right)$ distribution of the SM background process, and $x=m_{\ell \ell} / s^{1 / 2}$ with $s^{1 / 2}=13 \mathrm{TeV}$ being the center-of-mass energy of the proton collision. The fitting is performed in the region of $150 \mathrm{GeV}<m_{\ell \ell}<3000 \mathrm{GeV}$, and the result is shown in figure 4(a) and figure 4(b) for the dielectron and the dimuon channels, respectively. The background function used in eq. (3.1) is seen to fit the observed data very well.

We then perform the likelihood test of the "EWIMP signal + SM background" hypothesis by comparing the observational data shown in figure 4 with the following function:

$$
\frac{d N}{d m_{\ell \ell}}=\frac{d N_{\mathrm{BG}}}{d m_{\ell \ell}}+\frac{d N_{\mathrm{EWIMP}}}{d m_{\ell \ell}} .
$$

The likelihood is calculated in each bin of the lepton invariant mass based on the Poisson distribution, and maximize the total likelihood in the region of $150 \mathrm{GeV}<m_{\ell \ell}<$ $3000 \mathrm{GeV}$. The main contribution to the signal part, $d N_{\text {EWIMP }} / d m_{\ell \ell}$, comes from the interference between the LO (SM diagram) and the NLO (EWIMP one-loop diagram). In order to take into account the effect of k-factor, kinematical selection and so on, we calculate $d N_{\text {EWIMP }} / d m_{\ell \ell}$ by multiplying the factor $\left(\hat{\sigma}_{\mathrm{BSM}}-\hat{\sigma}_{\mathrm{SM}}\right) / \hat{\sigma}_{\mathrm{SM}}$ obtained in the previous section to the so-called $Z / \gamma^{*}$ background number estimated by the ATLAS collaboration [73] at each bin.

\subsection{MC based search}

The analysis is almost the same as the previous one, but the SM background is estimated by the Monte-Carlo (MC) simulation, as the ATLAS collaboration adopts. The current 


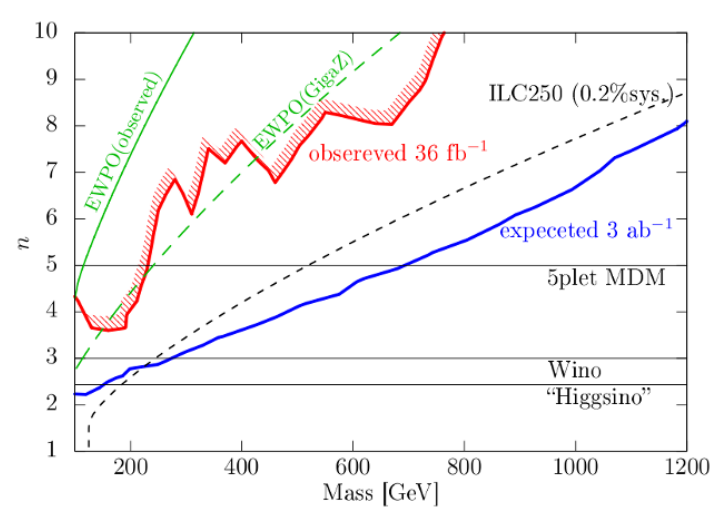

(a) Fitting based search

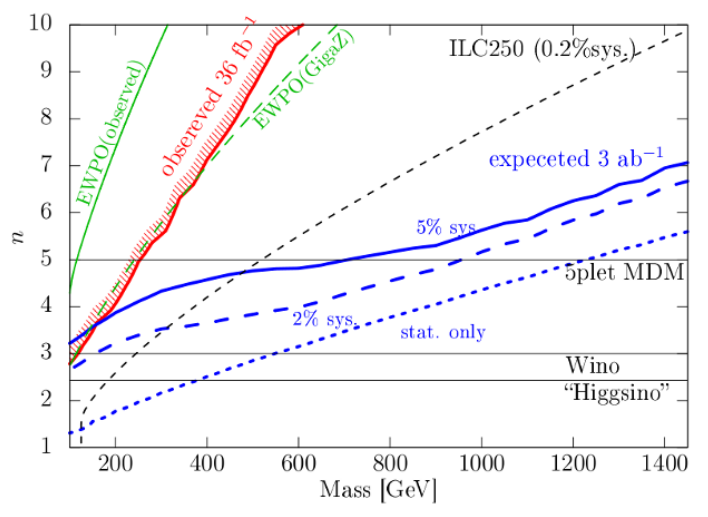

(b) MC based search

Figure 5. The constraint on a Majorana fermionic EWIMP at $95 \%$ confidence level obtained by the fitting based analysis (left panel) and the MC based analysis (right panel) on the plane of the EWIMP mass and the $\mathrm{SU}(2)_{\mathrm{L}}$ quantum number ' $\mathrm{n}$ '. The present constraint from $36 \mathrm{fb}^{-1}$ data at the $13 \mathrm{TeV}$ running is shown as a thick red line (associated with a small hatch) in both panels, while the future expected constraint from $3 \mathrm{ab}^{-1}$ data at the $14 \mathrm{TeV}$ running is shown as a blue solid line in the left panel and blue solid, dashed and dotted lines in the right panel, depending on the systematic uncertainty associated with the SM background estimation. Regions above the lines are (expected to be) excluded. Theoretical predictions from the well-motivated EWIMP candidates; wino, fermionic minimal dark matter (5plet MDM) and Higgsino, are also shown as horizontal (thin solid) black lines. On the other hand, the green solid line represents the constraint from the electroweak precision measurements, while the green dashed line is the future prospect assuming the electroweak precision measurements at the Giga-Z option. The future expected constraint from fermion pair productions at the international linear collider (ILC) with the $250 \mathrm{GeV}$ running is shown as a black dotted line.

systematic uncertainty is a few percent for $m_{\ell \ell} \lesssim 1 \mathrm{TeV}$. Using the MC based background and its systematic uncertainty given by the collaboration, we construct the likelihood for the "EWIMP signal + SM background" hypothesis, and put a constraint on the EWIMP. We assume that the systematic uncertainty at each bin is independent of others for simplicity.

\subsection{Capability of EWIMP detection at LHC}

We are now at the position to discuss the capability of the EWIMP detection at the LHC. Figure 5 shows the constraint on a Majorana fermionic EWIMP at 95\% confidence level. The $\mathrm{SU}(2)_{\mathrm{L}}$ quantum number ' $n$ ' is treated as a real number to depict the figure with the hypercharge of the EWIMP being zero. Three horizontal (thin solid) black lines are predictions from the well-motivated EWIMP candidates; wino, fermionic minimal dark matter (5plet MDM) and Higgsino. The $\mathrm{SU}(2)_{\mathrm{L}}$ quantum number of the Higgsino is estimated to be $n \simeq 2.43$, as it is not a Majorana fermion but a Dirac one. Since the effect of nonzero hypercharge on the Higgsino prediction is negligibly small, it is set to be zero. The constraint obtained by the "fitting based search" in section 3.1 is shown in the left panel (figure 5(a)), while that obtained by the "MC based search" in section 3.2 is shown in the right panel (figure $5(\mathrm{~b})$ ), respectively. 
The present constraint using $36 \mathrm{fb}^{-1}$ data at the $13 \mathrm{TeV}$ running is given by a thick red line (associated with a small hatch) in both panels. It can be seen that the fermionic minimal dark matter with the mass below about $250 \mathrm{GeV}$ is already excluded in both searches. We also consider the future prospect of the EWIMP detection at the HL-LHC assuming $3 \mathrm{ab}^{-1}$ data at the $14 \mathrm{TeV}$ running, ${ }^{3}$ which is given by a blue solid line in the left panel and blue solid, dashed and dotted lines in the right panel, as the future expected constraint at $95 \%$ confidence level. The systematic uncertainty in the MC based search is set to be $5 \%$ (blue solid line), $2 \%$ (blue dashed line) and $0 \%$ (blue dotted line), respectively.

In the fitting based analysis, it is possible to test Higgsino, wino and fermionic minimal dark matter with their masses up to $150 \mathrm{GeV}, 300 \mathrm{GeV}$, and $700 \mathrm{GeV}$, respectively. On the other hand, in the MC based analysis, it is in principle to test these well-motivated EWIMPs with their masses up to $380 \mathrm{GeV}, 550 \mathrm{GeV}$, and $1200 \mathrm{GeV}$, respectively. This result, of course, depends strongly on how well the systematic uncertainty (associated with the estimation of the SM background) is controlled. The use of the characteristic feature on the dilepton channels is actually very powerful. To make this fact clearer, we show the present constraint on the EWIMP from the electroweak precision measurements (combination of the four EWPOs in figure 3), which is shown as a green solid line in both panels. In addition, we also show the future prospects obtained by the EWPOs as a green dashed line, assuming the experimental uncertainties $\delta m_{W}=0.006 \mathrm{GeV}, \delta \Gamma_{Z}=0.8 \mathrm{MeV}$ and $\delta \sin ^{2} \theta_{\text {eff }}^{\ell}=0.0001$ at the Giga-Z option [75]. Moreover, we also show in both panels the future prospects of the EWIMP detection at the $250 \mathrm{GeV}$ international linear collider (ILC) as a black dotted line assuming the integrated luminosity of $2 \mathrm{ab}^{-1}$ and the beam polarizations of $\left(P_{-}=80 \%\right.$ and $\left.P_{+}:=30 \%\right)$. This prospect is obtained from the precision measurement of the dilepton process, $e^{-} e^{+} \rightarrow \mu^{-} \mu^{+}$, with the systematic uncertainty being $0.2 \%$ [76]. It can be seen that HL-LHC and ILC play a complementary role to search for the EWIMP; the HL-LHC has a good sensitivity for heavier EWIMPs, while the ILC has for lighter ones.

\section{Conclusion and discussion}

We have discussed in this paper the possibility of detecting EWIMP through the precision measurement of the dilepton (dielectron and dimuon) channels at the LHC. The EWIMP affects the lepton distribution of the channels through radiative corrections at $\mathcal{O}(1) \%$ level. Since detecting such a small deviation from the SM prediction is not trivial, we have considered two different methods to analyze data. It then turned out that both gives almost the same result. Moreover, the dilepton channels are comparable to and has a potential to be better than that of the mono- $X$ search with large energy to detect the EWIMP.

\footnotetext{
${ }^{3}$ In the fitting based search, the mock data is generated based on the fitting function (3.1), which is corrected by multiplying the ratio between $\hat{\sigma}_{\mathrm{SM}}$ at $\sqrt{s}=14 \mathrm{TeV}$ and $13 \mathrm{TeV}$. In the MC based analysis, the mock data is generated based on the background distribution estimated by ATLAS [73] instead of the fitting function.
} 
Though we focus mainly on non-colored EWIMPs which are an (almost) electroweak gauge eigenstate and has a tiny decay width, the method developed here is applicable to more generic EWIMPs. For instance, a neutralino or a chargino, which is described by a mixture of different electroweak gauge eigenstates, also provide the same effects on the Drell-Yan process. Another prominent example in the SM would be a top quark.

In order to make our analysis to be more accurate, we should take the following two issues into account: first, the correlation of systematic uncertainties among the bins of the lepton invariant mass should be included, which requires more detailed information about the estimation of the SM background. Next, the radiative correction from the EWIMP at around the threshold, namely $\hat{s} \sim 4 m^{2}$, receives a further correction by the so-called the threshold singularity when the electroweak quantum number of the EWIMP is large. Since we propose a novel idea to detect the EWIMP through the threshold observation, inclusion of these two issues is beyond our scope, and we leave it for a future work.

The effect of EWIMP scarcely affects the observables on the $Z$ pole as long as the EWIMP is much heavier than the $Z$ boson, as discussed in section 2. On the other hand, particularly when the EWIMP mass becomes comparable to the $Z$ boson mass, the effect should be involved to define all electroweak parameters consistently in the setup with the EWIMP, as we claim the measurement at the accuracy of $\mathcal{O}(1) \%$ [63]. The main contribution of the effect would be on the cross section of SM background processes. Although the size of the EWIMP signal will not be altered and thus our conclusion will keep unchanged, the above effects are eventually required to take into account for making the theoretical prediction of the EWIMP signal correctly at the accuracy of $\mathcal{O}(1) \%$. We leave this issue for a future work.

There are other interesting channels to detect the EWIMP at the LHC. For instance, observing the transverse mass distribution of a lepton and a missing energy (a neutrino) from the Drell-Yan process ( $s$-channel exchange of the $W$ boson) is also sensitive and expected to give a similar constraint on the EWIMP. An advantage of this channel is that its cross section is larger than those of the dilepton channels that we have developed in this paper.

Another interesting aspect of the threshold observation is the measurement of the EWIMP nature rather than the discovery. As seen in figure 2, the EWIMP correction depends strongly on its spin and electroweak quantum number. It would then be possible to pin down the EWIMP nature by measuring the Drell-Yan process precisely at hadron colliders.

\section{Acknowledgments}

S. Shirai thanks M. Endo and S. Mishima for useful discussion. This work is supported by Grant-in-Aid for Scientific Research from the Ministry of Education, Culture, Sports, Science, and Technology (MEXT), Japan, No. $17 \mathrm{H} 02878$ (S. M. and S. S.), 16H02176 (S. M. and M. T.), 26104009 (S. M.), 17H05399 (M. T.), 16H03991 (M. T.), and by World Premier International Research Center Initiative (WPI), MEXT, Japan. 
Open Access. This article is distributed under the terms of the Creative Commons Attribution License (CC-BY 4.0), which permits any use, distribution and reproduction in any medium, provided the original author(s) and source are credited.

\section{References}

[1] J. Hisano, S. Matsumoto and M.M. Nojiri, Explosive dark matter annihilation, Phys. Rev. Lett. 92 (2004) 031303 [hep-ph/0307216] [INSPIRE].

[2] J. Hisano, S. Matsumoto, M.M. Nojiri and O. Saito, Non-perturbative effect on dark matter annihilation and gamma ray signature from galactic center, Phys. Rev. D 71 (2005) 063528 [hep-ph/0412403] [INSPIRE].

[3] J. Hisano, S. Matsumoto, O. Saito and M. Senami, Heavy wino-like neutralino dark matter annihilation into antiparticles, Phys. Rev. D 73 (2006) 055004 [hep-ph/0511118] [INSPIRE].

[4] J. Hisano, K. Ishiwata and N. Nagata, A complete calculation for direct detection of Wino dark matter, Phys. Lett. B 690 (2010) 311 [arXiv:1004.4090] [INSPIRE].

[5] J. Hisano, K. Ishiwata and N. Nagata, Gluon contribution to the dark matter direct detection, Phys. Rev. D 82 (2010) 115007 [arXiv:1007.2601] [INSPIRE].

[6] J. Hisano, K. Ishiwata and N. Nagata, Direct Search of Dark Matter in High-Scale Supersymmetry, Phys. Rev. D 87 (2013) 035020 [arXiv:1210.5985] [INSPIRE].

[7] J. Hisano, K. Ishiwata and N. Nagata, QCD Effects on Direct Detection of Wino Dark Matter, JHEP 06 (2015) 097 [arXiv:1504.00915] [INSPIRE].

[8] L. Randall and R. Sundrum, Out of this world supersymmetry breaking, Nucl. Phys. B 557 (1999) 79 [hep-th/9810155] [INSPIRE].

[9] G.F. Giudice, M.A. Luty, H. Murayama and R. Rattazzi, Gaugino mass without singlets, JHEP 12 (1998) 027 [hep-ph/9810442] [INSPIRE].

[10] J.D. Wells, Implications of supersymmetry breaking with a little hierarchy between gauginos and scalars, in 11th International Conference on Supersymmetry and the Unification of Fundamental Interactions (SUSY 2003), Tucson U.S.A. (2003) [hep-ph/0306127] [INSPIRE].

[11] J.D. Wells, PeV-scale supersymmetry, Phys. Rev. D 71 (2005) 015013 [hep-ph/0411041] [INSPIRE].

[12] N. Arkani-Hamed and S. Dimopoulos, Supersymmetric unification without low energy supersymmetry and signatures for fine-tuning at the LHC, JHEP 06 (2005) 073 [hep-th/0405159] [INSPIRE].

[13] G.F. Giudice and A. Romanino, Split supersymmetry, Nucl. Phys. B 699 (2004) 65 [Erratum ibid. B 706 (2005) 487] [hep-ph/0406088] [INSPIRE].

[14] N. Arkani-Hamed, S. Dimopoulos, G.F. Giudice and A. Romanino, Aspects of split supersymmetry, Nucl. Phys. B 709 (2005) 3 [hep-ph/0409232] [INSPIRE].

[15] N. Arkani-Hamed, S. Dimopoulos and S. Kachru, Predictive landscapes and new physics at a $\mathrm{TeV}$, hep-th/0501082 [INSPIRE].

[16] ATLAS collaboration, Observation of a new particle in the search for the Standard Model Higgs boson with the ATLAS detector at the LHC, Phys. Lett. B 716 (2012) 1 [arXiv: 1207.7214] [INSPIRE]. 
[17] CMS collaboration, Observation of a new boson at a mass of $125 \mathrm{GeV}$ with the CMS experiment at the LHC, Phys. Lett. B 716 (2012) 30 [arXiv:1207.7235] [INSPIRE].

[18] L.J. Hall and Y. Nomura, Spread Supersymmetry, JHEP 01 (2012) 082 [arXiv:1111.4519] [INSPIRE].

[19] L.J. Hall, Y. Nomura and S. Shirai, Spread Supersymmetry with Wino LSP: Gluino and Dark Matter Signals, JHEP 01 (2013) 036 [arXiv:1210.2395] [INSPIRE].

[20] Y. Nomura and S. Shirai, Supersymmetry from Typicality: TeV-Scale Gauginos and PeV-Scale Squarks and Sleptons, Phys. Rev. Lett. 113 (2014) 111801 [arXiv:1407.3785] [INSPIRE].

[21] M. Ibe and T.T. Yanagida, The Lightest Higgs Boson Mass in Pure Gravity Mediation Model, Phys. Lett. B 709 (2012) 374 [arXiv:1112.2462] [INSPIRE].

[22] M. Ibe, S. Matsumoto and T.T. Yanagida, Pure Gravity Mediation with $m_{3 / 2}=10-100 \mathrm{TeV}$, Phys. Rev. D 85 (2012) 095011 [arXiv:1202.2253] [INSPIRE].

[23] A. Arvanitaki, N. Craig, S. Dimopoulos and G. Villadoro, Mini-Split, JHEP 02 (2013) 126 [arXiv:1210.0555] [INSPIRE].

[24] N. Arkani-Hamed, A. Gupta, D.E. Kaplan, N. Weiner and T. Zorawski, Simply Unnatural Supersymmetry, arXiv:1212.6971 [INSPIRE].

[25] T. Cohen, M. Lisanti, A. Pierce and T.R. Slatyer, Wino Dark Matter Under Siege, JCAP 10 (2013) 061 [arXiv:1307.4082] [INSPIRE].

[26] J. Fan and M. Reece, In Wino Veritas? Indirect Searches Shed Light on Neutralino Dark Matter, JHEP 10 (2013) 124 [arXiv:1307.4400] [INSPIRE].

[27] B. Bhattacherjee, M. Ibe, K. Ichikawa, S. Matsumoto and K. Nishiyama, Wino Dark Matter and Future dSph Observations, JHEP 07 (2014) 080 [arXiv: 1405.4914] [INSPIRE].

[28] M. Ibe, S. Matsumoto, S. Shirai and T.T. Yanagida, Wino Dark Matter in light of the AMS-02 2015 Data, Phys. Rev. D 91 (2015) 111701 [arXiv:1504.05554] [InSPIRE].

[29] M. Cirelli, N. Fornengo and A. Strumia, Minimal dark matter, Nucl. Phys. B 753 (2006) 178 [hep-ph/0512090] [INSPIRE].

[30] M. Cirelli, A. Strumia and M. Tamburini, Cosmology and Astrophysics of Minimal Dark Matter, Nucl. Phys. B 787 (2007) 152 [arXiv:0706.4071] [inSPIRE].

[31] M. Cirelli and A. Strumia, Minimal Dark Matter: Model and results, New J. Phys. 11 (2009) 105005 [arXiv: 0903.3381] [INSPIRE].

[32] R. Kitano and Y. Nomura, A Solution to the supersymmetric fine-tuning problem within the MSSM, Phys. Lett. B 631 (2005) 58 [hep-ph/0509039] [INSPIRE].

[33] H. Baer, V. Barger, P. Huang, A. Mustafayev and X. Tata, Radiative natural SUSY with a 125 GeV Higgs boson, Phys. Rev. Lett. 109 (2012) 161802 [arXiv:1207.3343] [INSPIRE].

[34] H. Baer, V. Barger and D. Mickelson, How conventional measures overestimate electroweak fine-tuning in supersymmetric theory, Phys. Rev. D 88 (2013) 095013 [arXiv:1309.2984] [INSPIRE].

[35] S. Gori, S. Jung and L.-T. Wang, Cornering electroweakinos at the LHC, JHEP 10 (2013) 191 [arXiv:1307.5952] [INSPIRE]. 
[36] C. Han, A. Kobakhidze, N. Liu, A. Saavedra, L. Wu and J.M. Yang, Probing Light Higgsinos in Natural SUSY from Monojet Signals at the LHC, JHEP 02 (2014) 049 [arXiv:1310.4274] [INSPIRE].

[37] Z. Han, G.D. Kribs, A. Martin and A. Menon, Hunting quasidegenerate Higgsinos, Phys. Rev. D 89 (2014) 075007 [arXiv: 1401.1235] [INSPIRE].

[38] J. Bramante, A. Delgado, F. Elahi, A. Martin and B. Ostdiek, Catching sparks from well-forged neutralinos, Phys. Rev. D 90 (2014) 095008 [arXiv: 1408.6530] [InSPIRE].

[39] H. Baer, A. Mustafayev and X. Tata, Monojet plus soft dilepton signal from light higgsino pair production at LHC14, Phys. Rev. D 90 (2014) 115007 [arXiv:1409.7058] [INSPIRE].

[40] J. Bramante et al., Relic Neutralino Surface at a 100 TeV Collider, Phys. Rev. D 91 (2015) 054015 [arXiv: 1412.4789] [inSPIRE].

[41] A. Ismail, E. Izaguirre and B. Shuve, Illuminating New Electroweak States at Hadron Colliders, Phys. Rev. D 94 (2016) 015001 [arXiv: 1605.00658] [INSPIRE].

[42] A. Freitas, S. Westhoff and J. Zupan, Integrating in the Higgs Portal to Fermion Dark Matter, JHEP 09 (2015) 015 [arXiv:1506.04149] [INSPIRE].

[43] A. Voigt and S. Westhoff, Virtual signatures of dark sectors in Higgs couplings, JHEP 11 (2017) 009 [arXiv: 1708.01614] [INSPIRE].

[44] M. Ibe, T. Moroi and T.T. Yanagida, Possible Signals of Wino LSP at the Large Hadron Collider, Phys. Lett. B 644 (2007) 355 [hep-ph/0610277] [INSPIRE].

[45] M.R. Buckley, L. Randall and B. Shuve, LHC Searches for Non-Chiral Weakly Charged Multiplets, JHEP 05 (2011) 097 [arXiv: 0909.4549] [INSPIRE].

[46] S. Asai, T. Moroi, K. Nishihara and T.T. Yanagida, Testing the Anomaly Mediation at the LHC, Phys. Lett. B 653 (2007) 81 [arXiv:0705.3086] [inSPIRE].

[47] S. Asai, T. Moroi and T.T. Yanagida, Test of Anomaly Mediation at the LHC, Phys. Lett. B 664 (2008) 185 [arXiv:0802.3725] [INSPIRE].

[48] S. Asai, Y. Azuma, O. Jinnouchi, T. Moroi, S. Shirai and T.T. Yanagida, Mass Measurement of the Decaying Bino at the LHC, Phys. Lett. B 672 (2009) 339 [arXiv:0807.4987] [INSPIRE].

[49] ATLAS collaboration, Search for long-lived charginos based on a disappearing-track signature in pp collisions at $\sqrt{s}=13 \mathrm{TeV}$ with the ATLAS detector, ATLAS-CONF-2017-017 (2017).

[50] M. Ibe, S. Matsumoto and R. Sato, Mass Splitting between Charged and Neutral Winos at Two-Loop Level, Phys. Lett. B 721 (2013) 252 [arXiv:1212.5989] [INSPIRE].

[51] J. McKay, P. Scott and P. Athron, Pitfalls of iterative pole mass calculation in electroweak multiplets, arXiv:1710.01511 [INSPIRE].

[52] R. Mahbubani, P. Schwaller and J. Zurita, Closing the window for compressed Dark Sectors with disappearing charged tracks, JHEP 06 (2017) 119 [Erratum ibid. 1710 (2017) 061] [arXiv: 1703.05327] [INSPIRE].

[53] H. Fukuda, N. Nagata, H. Otono and S. Shirai, Higgsino Dark Matter or Not: Role of Disappearing Track Searches at the LHC and Future Colliders, Phys. Lett. B 781 (2018) 306 [arXiv: 1703.09675] [INSPIRE].

[54] N. Arkani-Hamed, A. Delgado and G.F. Giudice, The Well-tempered neutralino, Nucl. Phys. B 741 (2006) 108 [hep-ph/0601041] [INSPIRE]. 
[55] K. Harigaya, K. Kaneta and S. Matsumoto, Gaugino coannihilations, Phys. Rev. D 89 (2014) 115021 [arXiv:1403.0715] [INSPIRE].

[56] A. Bharucha, F. Brümmer and R. Ruffault, Well-tempered n-plet dark matter, JHEP 09 (2017) 160 [arXiv:1703.00370] [INSPIRE].

[57] N. Nagata, H. Otono and S. Shirai, Probing Bino-Wino Coannihilation at the LHC, JHEP 10 (2015) 086 [arXiv: 1506. 08206] [inSPIRE].

[58] J. Hisano, D. Kobayashi, N. Mori and E. Senaha, Effective Interaction of Electroweak-Interacting Dark Matter with Higgs Boson and Its Phenomenology, Phys. Lett. B 742 (2015) 80 [arXiv:1410.3569] [INSPIRE].

[59] N. Nagata and S. Shirai, Higgsino Dark Matter in High-Scale Supersymmetry, JHEP 01 (2015) 029 [arXiv: 1410.4549] [INSPIRE].

[60] N. Nagata and S. Shirai, Electroweakly-Interacting Dirac Dark Matter, Phys. Rev. D 91 (2015) 055035 [arXiv: 1411.0752] [INSPIRE].

[61] D.S.M. Alves, J. Galloway, J.T. Ruderman and J.R. Walsh, Running Electroweak Couplings as a Probe of New Physics, JHEP 02 (2015) 007 [arXiv: 1410.6810] [INSPIRE].

[62] C. Gross, O. Lebedev and J.M. No, Drell-Yan constraints on new electroweak states: LHC as a $p p \rightarrow l^{+} l^{-}$precision machine, Mod. Phys. Lett. A 32 (2017) 1750094 [arXiv:1602.03877] [INSPIRE].

[63] M. Farina, G. Panico, D. Pappadopulo, J.T. Ruderman, R. Torre and A. Wulzer, Energy helps accuracy: electroweak precision tests at hadron colliders, Phys. Lett. B 772 (2017) 210 [arXiv: 1609.08157] [INSPIRE].

[64] M.E. Peskin and T. Takeuchi, Estimation of oblique electroweak corrections, Phys. Rev. D 46 (1992) 381 [INSPIRE].

[65] I. Maksymyk, C.P. Burgess and D. London, Beyond S, T and U, Phys. Rev. D 50 (1994) 529 [hep-ph/9306267] [INSPIRE].

[66] R. Barbieri, A. Pomarol, R. Rattazzi and A. Strumia, Electroweak symmetry breaking after LEP-1 and LEP-2, Nucl. Phys. B 703 (2004) 127 [hep-ph/0405040] [INSPIRE].

[67] S.P. Martin, K. Tobe and J.D. Wells, Virtual effects of light gauginos and higgsinos: A Precision electroweak analysis of split supersymmetry, Phys. Rev. D 71 (2005) 073014 [hep-ph/0412424] [INSPIRE].

[68] CDF, D0 collaboration, T.E.W. Group, 2012 Update of the Combination of CDF and D0 Results for the Mass of the W Boson, arXiv:1204.0042 [INSPIRE].

[69] ATLAS collaboration, Measurement of the $W$-boson mass in pp collisions at $\sqrt{s}=7 \mathrm{TeV}$ with the ATLAS detector, Eur. Phys. J. C 78 (2018) 110 [arXiv:1701.07240] [InSPIRE].

[70] Particle Data Group collaboration, C. Patrignani et al., Review of Particle Physics, Chin. Phys. C 40 (2016) 100001 [inSPIRE].

[71] Sld Electroweak Group, Delphi, Aleph, Sld, SLD Heavy Flavour Group, OPAL, LEP Electroweak Working Group and L3 collaborations, S. Schael et al., Precision electroweak measurements on the $Z$ resonance, Phys. Rept. 427 (2006) 257 [hep-ex/0509008] [INSPIRE]. 
[72] Delphi, OPAL, AlePh, LEP Electroweak Working Group and L3 collaborations, J. Alcaraz et al., A Combination of preliminary electroweak measurements and constraints on the standard model, hep-ex/0612034 [INSPIRE].

[73] ATLAS collaboration, Search for new high-mass phenomena in the dilepton final state using $36 \mathrm{fb}^{-1}$ of proton-proton collision data at $\sqrt{s}=13 \mathrm{TeV}$ with the ATLAS detector, JHEP 10 (2017) 182 [arXiv: 1707.02424] [INSPIRE].

[74] CDF collaboration, T. Aaltonen et al., Search for new particles decaying into dijets in proton-antiproton collisions at $\sqrt{s}=1.96 \mathrm{TeV}$, Phys. Rev. D 79 (2009) 112002 [arXiv: 0812.4036] [INSPIRE].

[75] H. Baer et al., The International Linear Collider Technical Design Report - Volume 2: Physics, arXiv:1306.6352 [INSPIRE].

[76] K. Harigaya, K. Ichikawa, A. Kundu, S. Matsumoto and S. Shirai, Indirect Probe of Electroweak-Interacting Particles at Future Lepton Colliders, JHEP 09 (2015) 105 [arXiv: 1504.03402] [INSPIRE]. 